\title{
ON UNIVERSAL SPACES OF THE TYPE $C(X)$
}

\section{H. ELTON LACEY AND PETER D. MORRIS}

In [1] Banach showed that every separable Banach space can be linearly isometrically embedded in $C[0,1]$. It is natural to ask for which compact Hausdorff space $X$ is it true that every separable Banach space can be linearly isometrically embedded in $C(X)$. A characterization of such $X$ 's is given in this paper when $C(X)$ is the real Banach space of all continuous real-valued functions on $X$. It is also shown that if $C(X)$ is not universal for all separable Banach spaces, then only a very restricted class of separable Banach spaces can be linearily isometrically embedded in $C(X)$. In particular, for each nonuniversal $C(X)$ with $\operatorname{dim} C(X)>1$ there is a two dimensional space which is not embeddable in $C(X)$. A characterization of those Banach spaces that can be linearly isometrically embedded in $C(X)$ for every infinite compact Hausdorff space $X$ is also given.

For a given Banach space $B, B^{\pi}$ denotes the closed unit sphere of the dual $B^{\prime}$ of $B$ in the weak* topology. The set of extreme points of $B^{\pi}$ is denoted by $E B^{\pi}$ and its weak ${ }^{*}$ closure is denoted by $\mathrm{cl}^{*} E B^{\pi}$. If $X$ is a compact Hausdorff space, $e$ denotes the evaluation map of $X$ in to $C(X)^{\prime}$. It is well known that $e$ is a homeomorphism into $C(X)^{\prime}$ with the weak ${ }^{*}$ topology and that $E C(X)^{\prime}=e(X) \cup-e(X)[2$, p. 86]. If $i: B \rightarrow C(X)$ is a linear isometry into, then the adjoint $i^{\prime}$ of $i$ maps $C(X)^{\pi}$ onto $B^{\pi}$, and, in fact, $i^{\prime}\left(E C(X)^{\pi}\right) \supseteq E B^{\pi}[3$, p. 441].

The following proposition shows that not all infinite compact spaces $X$ have the property that $C(X)$ is universal for the set of all separable Banach spaces.

1. Proposition. Let $B$ be a Banach space. Then $B$ is linearly, isometrically, embeddable in a $C(X)$ for $X$ dispersed if and only if $\mathrm{cl}^{*} E B^{\pi}$ is dispersed (see [6] for results on dispersed spaces, i.e., spaces without perfect subsets).

Proof. If $i: B \rightarrow C(X)$ is a linear isometry, then $i^{\prime}\left(E C(X)^{\pi}\right) \supseteq E B^{\pi}$. Hence, if $X$ is dispersed, then so is $E C(X)^{\pi}$ and thus, so is $\mathrm{cl}^{*} E B^{\pi}$. Conversely, if $\mathrm{cl}^{*} E B^{\pi}$ is dispersed, then $B$ is linearly, isometrically, embeddable in $C(X)$, where $X=\mathrm{cl}^{*} E B^{*}[2, \mathrm{p} .93]$.

In particular, since the two dimensional Euclidean space $B=l^{2}(2)$ has the property that $E B^{\pi}$ is not dispersed, it is not linearly and isometrically embeddable in $C(X)$ whenever $X$ is dispersed. It is now

Received by the editors February 6, 1967. 
shown that this property characterizes dispersed spaces and is also equivalent to the nonuniversality of $C(X)$.

2. Theorem. Let $X$ be a compact Hausdorff space. Then the following are equivalent.

(a) $X$ is dispersed,

(b) $C(X)$ contains no linear subspace $B$ of $\operatorname{dim}>1$ such that every element of norm 1 in $B^{\pi}$ is also in $E B^{\pi}$ (that is, $B^{\prime}$ is rotund),

(c) $C(X)$ contains no linear subspace $B$ of $\operatorname{dim}>1$ such that the unit sphere of $B$ is supported by only one hyperplane at each of its boundary points (that is, $B$ is smooth),

(d) $C(X)$ does not contain $l^{2}(2)$ linearly and isometrically,

(e) $C(X)$ is not universal for the set of all separable Banach spaces.

Proof. (a) implies (b). If $B \subseteq C(X)$ and $X$ is dispersed, then $\mathrm{cl}^{*} E B^{\pi}$ is dispersed. Hence, if $\operatorname{dim} B>1$, then $E B^{\pi}$ is not all of the boundary points of $B^{\pi}$.

(b) implies (c). Suppose $B \subseteq C(X), \operatorname{dim} B>1$, and $B$ is smooth. If $B_{1} \subseteq B$ is finite dimensional, $\operatorname{dim} B_{1}>1$, then $B_{1}$ is smooth and hence $B_{1}^{\prime}$ is rotund $[2$, p. 112].

(c) implies (d). $l^{2}(2)$ is smooth.

(d) implies (e). $l^{2}(2)$ is separable.

(e) implies (a). If $X$ is not dispersed, then there is a continuous function from $X$ onto $[0,1]$ (this is a simple modification of Urysohn's lemma to utilize perfectness, see $[6]$ also). Thus $C[0,1]$ is linearly and isometrically embeddable in $C(X)$.

A characterization of the Banach spaces that can be linearly and isometrically embedded in $C(X)$ for every infinite compact space $X$ is now given. The space $c$ is the Banach space of all real convergent sequences and $c_{0}$ is the space of all real sequences converging to 0 .

3. Theorem. Let $B$ be a Banach space. Then the following are equivalent.

(a) $B$ is linearly and isometrically embeddable in $C(X)$ for all infinite compact spaces $X$,

(b) $B$ is linearly and isometrically embeddable in $c$,

(c) $E B^{\pi}=Z \cup-Z$, where $Z$ is countable, $Z \cap-Z=\varnothing$, and $Z$ has at most one weak* limit point.

Proof. That (a) implies (b) is clear. (b) implies (c). Let $i$ be the linear isometry of $B$ into $c$. Then $\mathrm{cl}^{*} E B^{*} C i^{\prime}\left(E c^{\pi}\right)$ and $E c^{\pi}$ $=\{\delta n, \delta w,-\delta n,-\delta w\}$ where $\delta_{k}(j)=0$ if $k \neq j$ and $\delta_{k}(k)=1$ for $j, k=1, \cdots, w$. Also, $\delta_{k} \rightarrow \delta w$ in the weak ${ }^{*}$ topology. Let $k_{1}$ be the smallest integer such that $i^{\prime}\left(\delta_{k_{1}}\right) \in E B^{\pi}$ and let $Z_{1}=i^{\prime}\left(\delta k_{1}\right)$. Suppose 
$k_{1}<\cdots<k_{n}$ have been chosen so that $Z_{j}=i^{\prime}\left(\delta_{k_{j}}\right) \in E B^{\pi}, i^{\prime}\left(\delta_{j}\right)$ $\notin E B^{\pi}$ for $j \neq k_{i}, i=1, \cdots, n ; j<k n$, and $Z_{i} \neq-Z_{j}$ for $i, j$ $=1, \cdots, n$. If $\left\{ \pm Z_{j} \mid 1 \leqq j \in n\right\}=E B^{\pi}$, then $Z=\left\{Z_{1}, \cdots, Z_{n}\right\}$. If not, let $k_{n+1}$ be the smallest in teger such that $i^{\prime}\left(\delta_{k n+1}\right) \in E B^{\pi}, i^{\prime}\left(\delta_{k n+1}\right)$ $\neq \pm Z_{j}, j=1, \cdots, n$, and set $Z_{n+1}=i^{\prime}\left(\delta_{k n+1}\right)$. Thus, by induction one obtains a countable set $Z$ such that $Z \cap-Z=\varnothing, Z \cup-Z=E B^{x}$. Since $\left\{\delta_{k}\right\}$ has only one weak* limit point, $Z$ has a weak* limit point if and only if it is infinite, and, in such a case the limit point is $i^{\prime}(\delta w)$.

(c) implies (a). It is first shown that $B$ is linearly and isometrically embeddable in $c$, and then that $c$ is linearly and isometrically embeddable in $C(X)$. If $Z=\left\{Z_{1}, \cdots, Z_{n}\right\}$ then the map $i(x)(k)=\left\langle x, Z_{k}\right\rangle$ if $1 \leqq k \leqq n, i(x)(k)=0$ for $k>n$ is a linear isometry of $B$ into $c$ (actually into $\left.c_{0}\right)$. If $Z=\left\{Z_{1}, Z_{2}, \cdots\right\}$, then $\left\{Z_{n}\right\}$ converges in the weak* topology to some $Z_{w}$. The map $i(x)(k)=\left\langle x, Z_{k}\right\rangle$ for $1 \leqq k \leqq w$ is a linear isometry of $B$ into $c$. Now, if $X$ is not dispersed, then by (e) of Theorem 2, $C(X)$ is universal for all separable Banach spaces. Suppose $X$ is an infinite dispersed compact space. It suffices to show that there is a continuous map of $N=\{k \mid 1 \leqq k \leqq w\}$. It is clear that $X$ has infinitely many isolated points. Let $I=\{s n \mid n=1,2, \cdots\}$ be a countable collection of distinct isolated points of $X$. Define $h: X \rightarrow N$ by $h(s n)=n$ and $h(x)=w$ for all $x \in X \backslash I$. Since a subset of $N$ is closed if and only if it is either finite or contains $w$. Thus, the preimage under $h$ of a closed set is closed.

4. Corollary. $B$ is linearly and isometrically embeddable in $c_{0}$ if and only if $E B^{\pi}=Z \cup-Z$, where $Z \cap-Z=\varnothing, Z$ is either finite or the only weak* limit point of $Z$ is 0 .

Proof. If $i$ is the embedding of $B$ into $c_{0}$, then $E B^{\pi} \subset i^{\prime}\left(E c_{0}^{\pi}\right)$. Now, $E c_{0}^{\pi}=\{\delta n,-\delta n\}$ and $\delta n$ converges to 0 in weak ${ }^{*}$ topology. Thus, if $Z$ is chosen as in (b) implies (c) of Theorem 3, either $Z$ is finite or converges to 0 in the weak* topology.

The map defined in (c) implies (a) of Theorem 3 shows that if $E B^{*}$ $=Z \cup-Z, Z \cap-Z=\varnothing, Z$ is finite or converges to 0 in the weak* topology, then $B$ is embeddable in $c_{0}$.

EXAMPLE. There is a two dimensional space $B$ such that $E B^{\pi}$ is infinite and $B$ is linearly and isometrically embeddable in $c$. Let $\theta n=(n-1 / n)(\pi / 2)$ and $\theta w=\pi / 2$ and $\cup$ be the convex symmetric hull of $\{(\cos \theta k, \sin \theta k) \mid 1 \leqq k \leqq w\}$. Then $\cup$ is a closed, bounded, absorbing, symmetric, convex set and hence is the unit sphere of its Minkowski norm. If $A$ denotes this space and $B=A^{\prime}$, then $B$ is embeddable in $c$ by Theorem 3 . 
It is now shown that this is not true for $c_{0}$. Namely, a finite dimensional space $B$ is linearly and isometrically embeddable in $c_{0}$ if and only if $E B^{\pi}$ is finite.

5. Definition. A finite dimensional space $B$ is said to be polyhedral if its unit sphere has only a finite number of extreme points.

For results on polyhedral sets see [4] and [5]. The following proposition is given in $[2$, p. 266] also.

6. Proposition. Let $B$ be a finite dimensional Banach space. Then $B$ is polyhedral if and only if it is linearly and isometrically embeddable in $c_{0}$.

Proof. Suppose $B$ is polyhedral. Then so is $B^{\prime}[5$, p. 83]. Thus $E B^{\pi}$ is finite and by Corollary $4 B$ is embeddable in $c_{0}$. Conversely, if $B$ is linearly and isometrically embeddable in $c_{0}$, then $E B^{\pi}=Z \cup-Z$, where either $Z$ is finite or has 0 as its only weak* limit point. Since $B$ is finite dimensional, the latter case cannot happen. Hence $Z$ is finite, that is, $B^{\prime}$ is polyhedral. Thus $B^{\prime \prime}=B$ is polyhedral.

\section{REFERENCES}

1. Stephan Banach, Opérations linéaires, Chelsea, New York, 1955.

2. M. M. Day, Normed linear spaces, Academic Press, New York, 1962.

3. N. Dunford and J. T. Schwartz, Linear operators, Part I, Interscience, New York, 1958.

4. V. Klee, Polyhedral sections of convex bodies, Acta Math. 103 (1960), 243-267.

5. - Some characterizations of convex polyhedra, Acta Math. 102 (1959), 79-107.

6. A. Pelczynski, and Z. Semadeni, Spaces of continuous functions. III, Studia Math. 18 (1959), 210-222.

UNIVERSITY OF TEXAS AND

Pennsylvania State University 